\title{
Stabilization of Hypersonic Boundary Layers by Porous Coatings
}

\author{
Alexander V. Fedorov* \\ Moscow Institute of Physics and Technology, Zhukovski 140160, Russia \\ Norman D. Malmuth ${ }^{\dagger}$ \\ Rockwell Science Center, Thousand Oaks, California 91630 \\ and \\ Adam Rasheed ${ }^{\ddagger}$ and Hans G. Hornung ${ }^{\S}$ \\ California Institute of Technology, Pasadena, California 91125
}

\begin{abstract}
A second-mode stability analysis has been performed for a hypersonic boundary layer on a wall covered by a porous coating with equally spaced cylindrical blind microholes. Massive reduction of the second mode amplification is found to be due to the disturbance energy absorption by the porous layer. This stabilization effect was demonstrated by experiments recently conducted on a sharp cone in the T-5 high-enthalpy wind tunnel of the Graduate Aeronautical Laboratories of the California Institute of Technology. Their experimental confirmation of the theoretical predictions underscores the possibility that ultrasonically absorptive porous coatings may be exploited for passive laminar flow control on hypersonic vehicle surfaces.
\end{abstract}

\section{Nomenclature}

$\begin{array}{ll}A & =\text { admittance } \\ B & =\text { thermal admittance } \\ F & =\text { frequency parameter } \\ h & =\text { porous layer thickness } \\ n & =\text { porosity } \\ p & =\text { pressure perturbation } \\ P r & =\text { Prandtl number } \\ R e & =\text { displacement thickness Reynolds number } \\ R e_{\mathrm{tr}} & =\text { transition Reynolds number } \\ r & =\text { pore radius } \\ s & =\text { pore spacing } \\ T & =\text { mean flow temperature } \\ t & =\text { time } \\ U & =\text { mean flow velocity } \\ u, v, w & =\text { perturbation velocity components } \\ x, y, z & =\text { Cartesian coordinates } \\ \alpha, \beta & =\text { wavenumber components } \\ \gamma & =\text { specific heat ratio } \\ \delta^{*} & =\text { displacement thickness } \\ \theta & =\text { temperature perturbation } \\ \kappa & =\text { thermal conductivity } \\ \mu & =\text { viscosity } \\ \rho & =\text { mean flow density } \\ \sigma & =\text { spatial growth rate } \\ \varphi & =\text { wave front angle } \\ \omega & =\text { angular frequency } \\ & \end{array}$

Subscripts

$=$ upper boundary-layeredge

$m \quad=$ maximum value

$w \quad=$ wall surface

Superscript

$=$ dimensional

Received 11 February 2000; revision received 28 August 2000; accepted for publication 3 October 2000; presented as Paper 2001-0891 at the AIAA 39th Aerospace Sciences Meeting, Reno, NV, 8-11 January 2001. Copyright (C) 2000 by the authors. Published by the American Institute of Aeronautics and Astronautics, Inc., with permission.

* Associate Professor, Department of Aeromechanics and FlightEngineering. Member AIAA.

${ }^{\top}$ Senior Scientist, Fluid Dynamics. Fellow AIAA.

$\$$ Ph.D. Student, Graduate Aeronautical Laboratories.

${ }^{\S}$ Director, Graduate Aeronautical Laboratories.

\section{Introduction}

$\mathbf{T}$ HE ability to stabilize a hypersonic boundarylayer and increase its laminar run is of critical importance in the hypersonic vehicle design. ${ }^{1}$ Early transition causes significant increases in heat transfer and skin friction. Higher heating requires an increased performance thermal protection system (TPS), active cooling, or trajectory modification. This translates to higher cost and weight of hypersonic vehicles due to increased TPS weight. Moreover, with the low payload mass fraction, even small savings in TPS weight can provide a significant payload increase. Vehicle maintainability and operability are also affected by transition. Robust metallic TPS have temperature limits lower than ceramic TPS. Laminar flow control (LFC) can help meet these more severe constraints. For a streamlined vehicle with large wetted area, viscous drag becomes important. It can be from $10 \%$ (fully laminar) to $30 \%$ (fully turbulent) of the overall drag. ${ }^{2}$ For optimized hypersonic wave/riders, viscous drag may represent up to $50 \%$ of the total drag. ${ }^{3}$ Vehicle aerodynamics is another area impacted by laminar-turbulent transition. Asymmetry of the transition locus can produce significant yawing moments. Aerodynamic control surfaces and reaction control systems are also affected due to sensitivity of boundary-layer separation to the flow state (laminar or turbulent).

If freestream disturbances and TPS-induced perturbations are small, transition to turbulence is due to amplification of unstable boundary-layer modes. ${ }^{1,4}$ In this case, LFC methods and transition prediction tools are predominantly based on stability theory and experiment. $^{5-8}$ LFC systems are aimed at slowing down or eliminating amplification of unstable disturbances using passive and/or active control techniques. A third form of flow control is known as reactive control, in which boundary-layer disturbances are canceled by artificially introducing out-of-phase disturbances. Typical passive LFC techniques are pressure gradient and shaping. Active techniques include wall suction and heat transfer. In reactive control methods, periodic suction/blowing, heating/cooling or wall vibrations are used for artificial excitation of counter-phasedisturbances.

In hypersonic boundary layers, amplification of the following instability mechanisms may drive the transition process:

1) The first instability mechanism is the first mode associated with Tollmien-Schlichting waves. This instability may be dominant at relatively small local Mach numbers (normally less than 5). This mode is strongly stabilized on cool surfaces. At low walltemperature ratios, the stabilization effect may be so strong that the first-mode mechanism becomes unimportant.

2) The second mode associated with an inviscid instability present due to a region of supersonic mean flow relative to the disturbance phase velocity ${ }^{5}$ belongs to the family of trapped acoustic modes and becomes the dominant instability in two-dimensionaland 


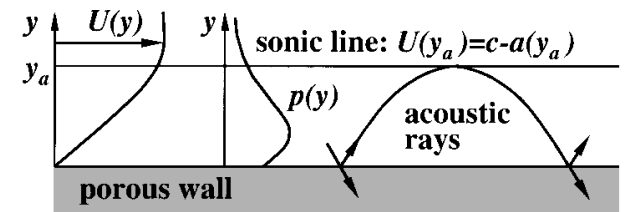

Fig. 1 Acoustic mode in a supersonic boundary layer on semitransparent wall.

quasi-two-dimensional boundary layers at Mach numbers $M>4$. The existence of the second mode was established by the experiments of Kendall, ${ }^{9}$ Demetriades, ${ }^{10}$ Stetson et al., ${ }^{11}$ Stetson and Kimmel, ${ }^{12}$ and Kimmel et al. ${ }^{13}$ The most amplified second-mode wavelength is approximately twice the boundary-layer thickness, and its phase velocity tends to the boundary-layeredge velocity of mean flow. As a result, the second-mode disturbances are in the ultrasonic frequency band. For example, the most amplified waves observed in the experiment of Stetson et al. ${ }^{11}$ at Mach 8 correspond to a frequency about $100 \mathrm{kHz}$. In contrast to the first mode, the second mode is destabilized by cooling.

3) Crossflow vortices are observed in three-dimensionalboundary layers on the leading edge of a swept wing, axisymmetric bodies at high angles of attack, etc. This instability is weakly sensitive to wall cooling. It can be effectively stabilized by shaping. For example, two-dimensional shaping of air breathers helps to avoid crossflow instabilities on large acreage regions of the vehicle surface.

4) Görtler vortices play a major role in transition on concave surfaces. Similar to the crossflow instability, their growth rate can be reduced by shaping.

Because severe environmental conditions make it difficult to use active and reactive LFC concepts for hypersonic vehicles, passive LFC techniques are of great interest. Thus, Malmuth et al. ${ }^{14}$ proposed a new passive method of second- and higher-mode stabilization. They exploited the hypersonic boundary layer's behavior as an acoustic waveguide, schematically shown in Fig. 1. Therein, acoustic rays are reflected by the wall and turn around near the sonic line: $y=y_{a}, U\left(y_{a}\right)=\operatorname{Re}(c)-a\left(y_{a}\right)$, where $c$ is the disturbance phase speed and $a$ is local sound speed. The second, third, and higher boundary-layermodes correspond to the waveguide normal modes. Malmuth et al. ${ }^{14}$ assumed that the absorption of acoustic energy by an ultrasonically absorptive coating can stabilize these disturbances. This assumption was examined using stability theory for inviscid disturbances. It was found that an ultrasonically semitransparent wall provides substantial reduction of the second-mode growth rate.

In this paper, we study this stabilization mechanism, including viscous effects and an absorptive skin microstructure. We formulate the eigenvalue problem for viscous disturbances in a hypersonic boundary layer on a wall covered by an ultrasonically absorptive coating of special type, namely, a porous layer with equally spaced cylindrical blind microholes. We obtain the analytical form of boundary conditions on the porous surface and solve the viscous eigenvalue problem numerically. We discuss results of calculations showing the second-mode stabilization on porous surfaces of various pore radii, spacing, and thickness. Then we briefly describe the experimental data of Rasheed et al. ${ }^{15}$ that confirm the theoretically based hypersonic boundary-layer stabilization by porous coatings given in this paper. These results were obtained in the T-5 Graduate Aeronautical Laboratories of the California Institute of Technology high-enthalpy wind tunnel on a sharp cone that they detail in Ref. 15. Finally, we conclude the paper with a summary discussion and indicate possible future directions.

\section{Eigenvalue Problem}

We consider supersonic boundary-layer flow over a flat plate or sharp cone as schematically shown in Fig. 2. The fluid is a perfect gas with Prandtl number $P r$, specific heat ratio $\gamma$, and viscosity $\mu$. The coordinates $x, y$, and $z$ are made nondimensionless using the boundary-layer displacement thickness $\delta^{*}$. In the locally parallel approximation, the mean flow is characterized by the profiles of $x$-component velocity $U(y)$ and temperature $T(y)$, referenced to the quantities $U_{e}^{*}$ and $T_{e}^{*}$ at the upper boundary-layeredge. Three-

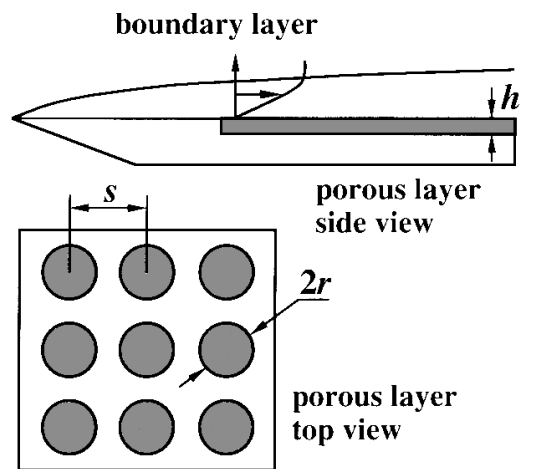

Fig. 2 Schematic of a wall covered by porous layer.

dimensional disturbances are represented in the traveling wave form

$$
\tilde{q}=\operatorname{Re}\{q(y) \exp i(\alpha x+\beta z-\omega t)]\}, \quad \tilde{q}=\tilde{u}, \tilde{v}, \tilde{w}, \tilde{p}, \tilde{\theta}]
$$

where $\tilde{u}, \tilde{v}$, and $\tilde{w}$ are velocity components; $\tilde{p}$ is the pressure referenced to the double dynamic pressure $\rho_{e}^{*} U_{e}^{* 2} ; \tilde{\theta}$ is the temperature; $\alpha=\alpha^{*} \delta^{*}$ and $\beta=\beta^{*} \delta^{*}$ are wave number components; and $\omega=\omega^{*} \delta^{*} / U_{e}^{*}$ is the angularfrequency. The system of stability equations that is derived from the full Navier-Stokes equations for a locally parallel compressible boundary layer can be represented in the form ${ }^{16}$

$$
\frac{\mathrm{d} z}{\mathrm{~d} y}=S \cdot z, \quad z=\left(u, \frac{\mathrm{d} u}{\mathrm{~d} y}, v, p, \theta, \frac{\mathrm{d} \theta}{\mathrm{d} y}, w, \frac{\mathrm{d} w}{\mathrm{~d} y}\right)^{T}
$$

where $S$ is an $8 \times 8$ matrix. Its elements are functions of the mean flow profiles, the displacement thickness Reynolds number $R e=$ $\delta^{*} U_{e}^{*} \rho_{e}^{*} / \mu_{e}^{*}$, and disturbance characteristics $\omega, \alpha$, and $\beta$.

We consider a wall covered by a porous layer of the thickness $h^{*}$. The pores are equally spaced cylindricalblind holes of radius $r^{*}$ perpendicular to the wall surface, as schematically shown in Fig. 2. The hole spacing $s^{*}$ and diameter are assumed to be much less than the boundary-layerdisplacement thickness $\delta^{*}$. Because the pore radius is small and interactions between neighboring pores are weak, perturbations of longitudinal and transverse velocity produced by the porous layer are neglected. However, the porous structure is semitransparentrelative to the vertical velocity and temperature perturbations. In this case, the wall boundary conditions can be expressed as
$u(0)=0$,
$w(0)=0$
$v(0)=A p(0)$
$\theta(0)=B p(0)$

where the admittance $A$ and thermal admittance $B$ are complex quantities that depend on properties of the wall material, porosity parameters, mean flow characteristics on the wall surface, and flow perturbation parameters such as a wave frequency and wavelength. These dependencies are derived in the next section. Because boundary-layer modes decay outside the boundary layer, we have

$$
u(\infty)=v(\infty)=w(\infty)=\theta(\infty)=0
$$

The eigenvalue problem (2-4) provides the dispersion relation $F(\alpha, \beta, \omega)=0$. For temporal stability, the wave number components $\alpha$ and $\beta$ are real quantities, and $\omega$ is a complex eigenvalue. If $\operatorname{Im}(\omega)>0$, then the disturbance is unstable. For spatial instability in two-dimensional boundary layers, the frequency $\omega$ and transverse wave number component $\beta$ are real, whereas $\alpha$ is a complex eigenvalue. If $\operatorname{Im}(\alpha)<0$, then the disturbance amplifies downstream with the spatial growth rate $\sigma=-\operatorname{Im}(\alpha)$

\section{Admittance of Porous Layer}

The porous layer is characterized by the porosity $n$, which is the fraction of the overall volume taken up by the pores. For the pore spacing shown in Fig. 2, the porosity, $n=\pi\left(r^{*} / s^{*}\right)^{2}$, can be varied in the range $0<r^{*} / s^{*}<\pi / 4$, where the upper limit corresponds to $s^{*}=2 r^{*}$. The pore radius and spacing are considered to be much less than the disturbance wavelength, which is of the order of the 
boundary-layer displacement thickness. In this case, the porosity is fine enough to avoid disturbing the laminar boundary layer by other mechanisms associated with effective surface roughness. The porous layer thickness $h^{*}$ is assumed to be much larger than the pore radius $r^{*}$, that is, each pore is treated as a long tube.

To obtain the relationship between the admittance $A$ and porous layer parameters, we use the theoretical model developed by Gaponov for subsonic ${ }^{17,18}$ and moderate supersonic speeds. ${ }^{19}$ These studies addressed the porosity effect on Tollmien-Schlichting (TS) waves. As contrasted to second-mode waves of acoustic type discussed in this paper, the TS waves over porous walls analyzed by Gaponov are vortical disturbances that become unstable due to viscous mechanisms. For this reason, the second-modeinteraction with a porous surface is fundamentally different from that of TS waves. Yet, the results ${ }^{18}$ for the disturbance propagation within a porous wall are independent of the nature of the boundary-layer disturbances, for example, second-mode acoustic or TS waves. In particular, they can be used in formulating the porous wall boundary conditions for the vertical velocity of second-mode disturbances considered herein. The thermal admittance $B$ is derived using an explicit coupling between the pressure, temperature, and velocity perturbations within a uniform pore..$^{20}$

Following the analysis, ${ }^{18}$ we apply the theory of sound wave propagation in thin and long tubes (see, for example, Ref. 21). Because $h^{*} \gg r^{*}$, the pressure is approximately constant across the pore. In this case, the acoustic field within each pore is characterized by the propagation constant $\Lambda$ and the characteristicimpedance $Z_{0}$. These parameters can be expressed as a function of the series impedance $Z$ and the shunt admittance $Y$ for the tube element of unit length using the transmission line formalism. ${ }^{22,23}$ The series impedance properties of the tube element are associated with the storage of kinetic energy and its dissipation due to viscous losses at the tube wall. The shunt admittance is associated with the potentialenergy of compression and the thermal energy losses due to the wall heat conductivity. We assume that the mean gas temperature along the tube is constant and equal to the wall surface temperature $T_{w}$. Daniels ${ }^{22}$ and Benade ${ }^{23}$ showed that the dimensional series impedance $Z^{*}$ and shunt admittance $Y^{*}$ per unit length of a tube with radius $r^{*}$ are expressed as

$$
\begin{gathered}
Z^{*}=-\frac{i \omega^{*} \rho_{w}^{*}}{\pi r^{* 2}}\left[1-\frac{2}{k_{v}} \cdot \frac{J_{1}\left(k_{v}\right)}{J_{0}\left(k_{v}\right)}\right]^{-1} \\
Y^{*}=-\frac{i \omega^{*} \pi r^{* 2}}{\rho_{w}^{*} a_{w}^{* 2}}\left[1+(\gamma-1) \frac{2}{k_{t}} \cdot \frac{J_{1}\left(k_{t}\right)}{J_{0}\left(k_{t}\right)}\right]
\end{gathered}
$$

where, $\rho_{w}^{*}$ and $a_{w}^{*}$ are mean density and sound speed in a tube. $J_{0}$ and $J_{1}$ are Bessel functions of the arguments $k_{v}=r^{*} \sqrt{ }\left(i \omega^{*} \rho_{w}^{*} / \mu_{w}^{*}\right)$ and $k_{t}=k_{v} \sqrt{ }(P r)$, which measure the ratio of the tube radius to the viscous boundary-layerthickness and to the thermal boundary-layer thickness on the tube surface, respectively. Using the relation

$$
J_{0}(x)+J_{2}(x)=2 J_{1}(x) / x
$$

we express $Z^{*}$ and $Y^{*}$ in the form

$$
\begin{gathered}
Z^{*}=\frac{i \omega^{*} \rho_{w}^{*}}{\pi r^{* 2}} \cdot \frac{J_{0}\left(k_{v}\right)}{J_{2}\left(k_{v}\right)} \\
Y^{*}=-\frac{i \omega^{*} \pi r^{* 2}}{\rho_{w}^{*} a_{w}^{* 2}}\left[\gamma+(\gamma-1) \frac{J_{2}\left(k_{t}\right)}{J_{0}\left(k_{t}\right)}\right]
\end{gathered}
$$

For the average velocity through the pore, the transmission line is characterized by the impedance $Z_{1}^{*}=S^{*} Z^{*}$ and shunt admittance $Y_{1}^{*}=Y^{*} / S^{*}$, where $S^{*}=\pi r^{* 2}$ is pore cross-sectionalarea. Choosing the boundary-layerdisplacement thickness and mean flow parameters at the upper boundary-layeredge as reference scales, we have

$$
\begin{gathered}
Z_{1} \equiv \frac{\pi r^{* 2} \delta^{*}}{\rho_{e}^{*} U_{e}^{*}} Z^{*}=\frac{i \omega}{T_{w}} \frac{J_{0}\left(k_{v}\right)}{J_{2}\left(k_{v}\right)}, \quad k_{v}=r \sqrt{\frac{i \omega \rho_{w}}{\mu_{w}} R} \\
Y_{1} \equiv \frac{\rho_{e}^{*} U_{e}^{*} \delta^{*}}{\pi r^{* 2}} Y^{*}=-i \omega M^{2}\left[\gamma+(\gamma-1) \frac{J_{2}\left(k_{t}\right)}{J_{0}\left(k_{t}\right)}\right]
\end{gathered}
$$

where $r=r^{*} / \delta^{*}$ is nondimensional pore radius. The characteristic impedance $Z_{0}$ and the propagation constant $\Lambda$ are expressed in the form

$$
Z_{0}=\sqrt{Z_{1} / Y_{1}}, \quad \Lambda=\sqrt{Z_{1} Y_{1}}, \quad \operatorname{Re}(\Lambda)<0
$$

The coupling between the pressure amplitude $p$ and the average velocity disturbance amplitude $\hat{v}$ at the pore end, $y=-h$, can be expressed as $p(-h)=X \cdot \hat{v}(-h)$, where the impedance $X$ depends on characteristics of the backup structure. If the lower pore end is closed by a solid wall (blind pores), then $\hat{v}(-h)=0$. In this case, the impedance is $X=\infty$, and the velocity-pressure ratio at the upper end of the pore is

$$
\hat{v}(0) / p(0)]=\left(1 / Z_{0}\right) \tanh (\Lambda h)
$$

Averaging the vertical velocity amplitude at the wall over the surface area, we have $v(0)=n \cdot \hat{v}(0)$. Then the admittance in the boundary conditions (3) is expressed as

$$
A=\left(n / Z_{0}\right) \tanh (\Lambda h)
$$

If the porous layer is relatively thick $(\Lambda h \rightarrow \infty)$, then Eq. (14) is reduced to the form

$$
A=-\left(n / Z_{0}\right)
$$

Note that the limit $\Lambda h \rightarrow \infty$ leads to Eq. (15) at any finite value of $X$ (i.e., the disturbance at the upper end of each hole does not feel the lower end due to the decay of sound propagating along a tube).

According to the analysis of Stinson and Champoux,${ }^{20}$ the pressure disturbance, average temperature disturbance, and average velocity disturbance within a cylindrical pore are coupled as

$$
\begin{gathered}
\hat{v}^{*}\left(\omega^{*}\right)=\frac{1}{i \omega^{*} \rho_{w}^{*}} \frac{\mathrm{d} p^{*}}{\mathrm{~d} y^{*}}\left(\omega^{*}\right)\left[1-\frac{2}{k_{v}} \frac{J_{1}\left(k_{v}\right)}{J_{0}\left(k_{v}\right)}\right] \\
\hat{\theta}^{*}\left(\omega^{*}\right)=\frac{i \omega^{*} \mu^{*}}{\kappa_{w}^{*}}\left[p^{*}\left(\omega^{*}\right) / \frac{\mathrm{d} p^{*}}{\mathrm{~d} y^{*}}\left(\operatorname{Pr} \omega^{*}\right)\right] \hat{v}^{*}\left(\operatorname{Pr} \omega^{*}\right)
\end{gathered}
$$

SubstitutingEq. (16) into Eq. (17), accountingfor Eq. (7), and nondimensionalizng the result, we obtain

$$
\hat{\theta}=-(\gamma-1) M^{2} T_{w} p J_{2}\left(k_{t}\right) / J_{0}\left(k_{t}\right)
$$

Thus, the thermal admittance in the boundary condition (3) for the temperature disturbance is expressed as

$$
B=-n(\gamma-1) M^{2} T_{w} J_{2}\left(k_{t}\right) / J_{0}\left(k_{t}\right)
$$

\section{Computational and Parametric Studies}

To evaluate the porous layer effect on the second-mode stability, we solve the eigenvalue problem (2-4) numerically using the admittance (14) or its limiting form (15) and the thermal admittance (19). We consider the boundary layer of a perfect gas with Prandtl number $\operatorname{Pr}=0.71$ and specific heat ratio $\gamma=1.4$. The temperatureviscosity law is specified as $\mu=\mu_{0}\left(T / T_{0}\right)^{m}$ with the exponent $m=0.75$; the second viscosity is zero. Figure 3 shows the spatial growth rate $\sigma$ as a function of the Reynolds number $R e$ for two-dimensional unstable waves $(\beta=0)$ of nondimensional frequency $F \equiv \omega^{*} v_{e}^{*} / U_{e}^{* 2}=2.8 \times 10^{-4}$ in the boundary layer at the Mach number $M=6$. The wall temperature $T_{w}=1.4$ approximately corresponds to the wall temperature ratio $T_{w} / T_{\mathrm{ad}}=0.2$. Calculations were conducted for a thick porous layer $(\Lambda h \rightarrow \infty)$ with the porosity $n=0.5$ at various values of the nondimensionalpore radius $r=r^{*} / \delta^{*}$. Note that the porous layer causes massive reduction of the second-mode growth rate. In Figs. 3-6, symbols correspond to the case of zero thermal admittance, $B=0$. For all cases considered, temperature perturbations on the porous surface weakly affect the disturbance growth rate and can be neglected.

Figure 4 shows that deep pores of fixed radius $(r=0.03$ at $R e=2 \times 10^{3}$ ) and spacing (porosity $n=0.5$ ) strongly stabilize the second-mode waves in a wide frequency band at various Reynolds numbers $R e$ (dashed lines). This example illustrates that it is possible to cause significant reduction of the disturbance growth rate on large surface areas without fine tuning the pore size. As contrasted to 


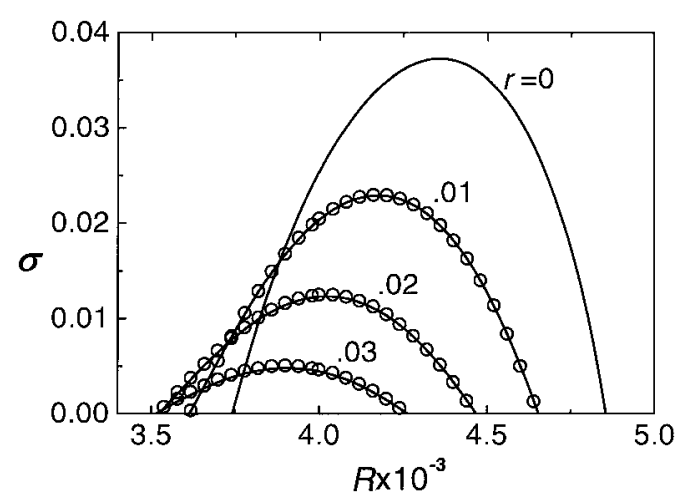

Fig. 3 Growth rate $\sigma$ as a function of Reynolds number $R e$ at various pore radii $r: M=6, T_{w}=1.4, F=2.8 \times 10^{-4}, n=0.5$, and $\Lambda h \rightarrow \infty$ (solid lines); symbols indicate zero thermal admittance. $(R=$ Reynolds number in figure.)

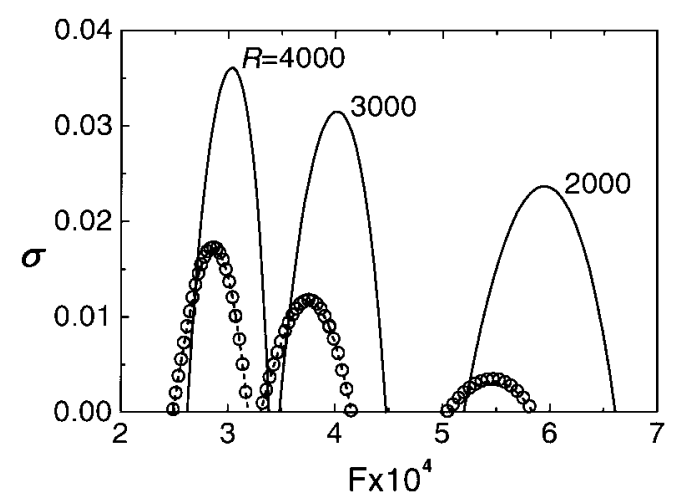

Fig. 4 Growth rate $\sigma$ as a function of disturbance frequency $F$ at various Reynolds numbers $R e: M=6, T_{w}=1.4, n=0.5$, and $\Lambda h \rightarrow \infty$; solid lines $r=0$, dashed lines $r=0.03$ at $R e=2.0 \times 10^{3}$, symbols indicate zero thermal admittance. $(R=$ Reynolds number in figure. $)$

reactive flow control techniques, a porous coating provides passive stabilization of the boundary-layer flow regardless the disturbance phase and amplitude distributions in space and time and with no external energy input. Note that the waveguide behavior described earlier in this paper in connection with the instability of the acoustic second mode that is quenched by the ultrasonic absorbing wall concept described herein resembles amplification processes studied by the second author in connection with the stability of hypersonic strong interaction flows. ${ }^{24}$

Figure 5 shows distributions of the maximum growth rate, $\left.\sigma_{m}(R e)=\max _{\omega} \sigma(\omega, R e)\right]$, at the wall temperatures $T_{w}=1.4,3.5$, and 7.0, that approximately corresponds to the wall temperature ratio $T_{w} / T_{\text {ad }}=0.2,0.5$, and 1 . The stabilization effect decreases as the wall temperature increases. A strong reduction of the growth rate is observed in the boundary layer on a cool wall (see Fig. 5), a more practical case for hypersonic applications. This trend is consistent with the admittance asymptotic behavior associated with Eqs. (10$12)$ and (15). For deep pores $(\Lambda h \gg 1)$ of relatively small radius $\left(\left|k_{v}\right| \ll 1\right)$, the admittance $A$ is proportional to $k_{v} M \sqrt{ }\left(T_{w}\right)$ and decreases with the wall temperature as $T_{w}^{-m / 2}$.

Figure 6 shows the maximum growth rate $\sigma_{m}$ as a function of the porosity $n$ for $R e=4 \times 10^{3}$ and $r=0.03$ for the boundary layer at $M=6$ and $T_{w}=1.4$. The porous layer of spacing $s=4 r(n \approx 0.2)$ reduces the growth rate by a factor of 2 compared to the solid wall case $n=0$. Our calculations using the $e^{N}$ method indicates that this stabilization translates to extending the transition onset point more than three times its value without porosity. In Fig. 7, the second-mode growth rate is shown as a function of the nondimensional porous layer thickness $h=h^{*} / \delta^{*}$ at $n=0.4, r=0.03$, $R e=4 \times 10^{3}$, and $F=3 \times 10^{-4}$. The limit $\Lambda h \rightarrow \infty$ is achieved at a relatively small value of $h \approx 0.3$ (pore depth is about five diameters) that is due to strong damping of sound waves in thin pores. There is an optimal thickness, $h \approx 0.12$, at which the porous
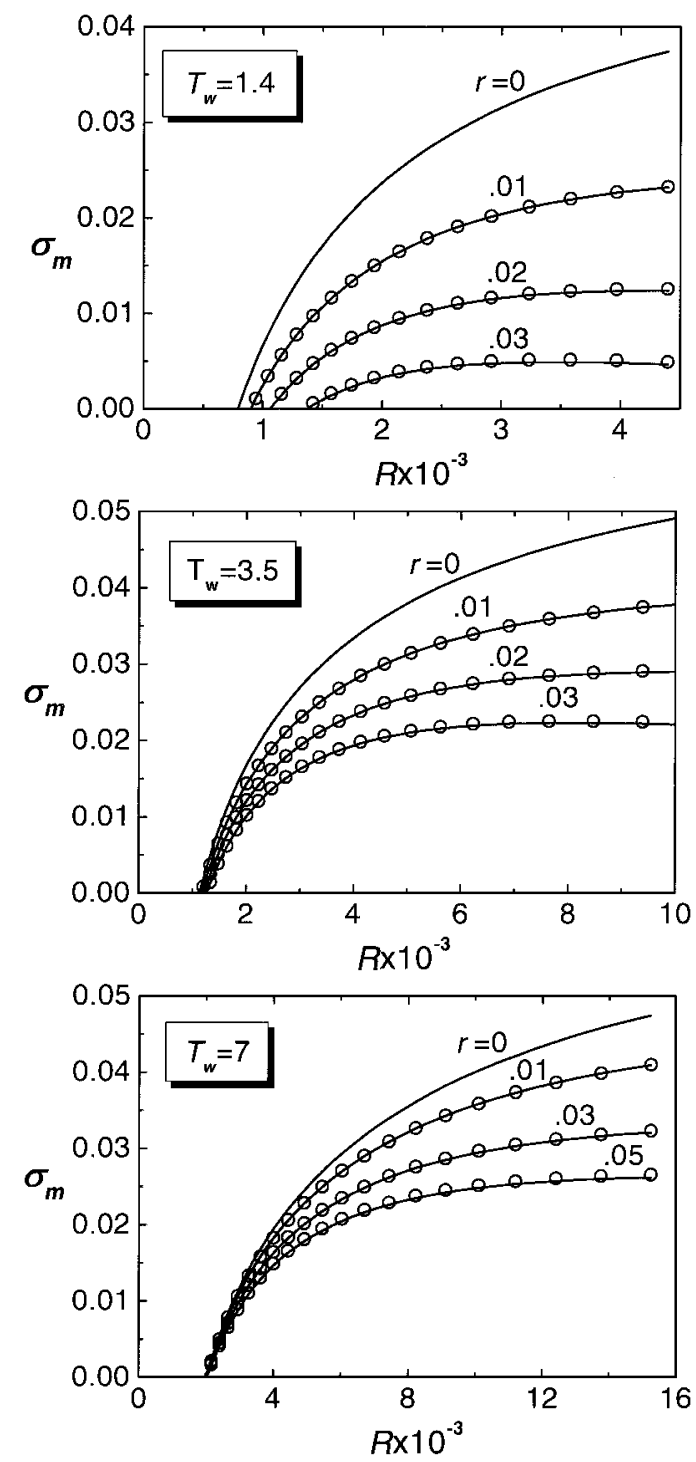

Fig. 5 Distributions of maximum growth rate $\sigma_{m}(R e)$ at various pore radii $r: M=6, n=0.5$, and $\Lambda h \rightarrow \infty(-)$; symbols indicate zero thermal admittance. $(R=$ Reynolds number in figure. $)$

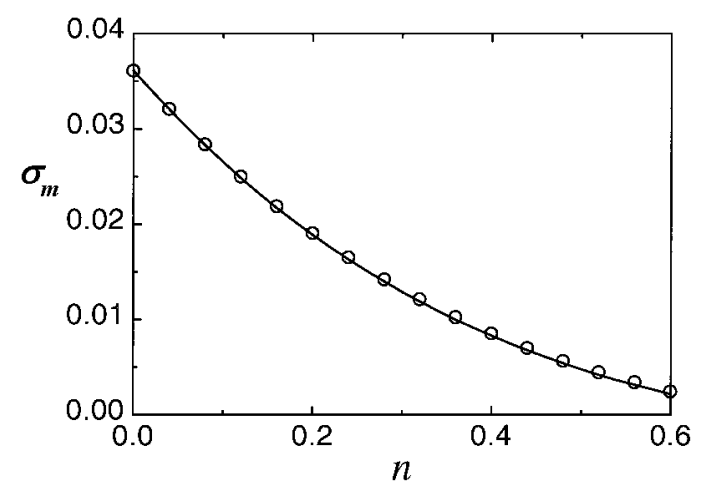

Fig. 6 Maximum growth rate $\sigma_{m}$ as a function of porosity $n$ at $R e=4 \times 10^{3}: M=6, T_{w}=1.4, r=0.03$, and $\Lambda h \rightarrow \infty$ (—); symbols indicate zero thermal admittance.

wall effect is able to stabilize the disturbance completely. In this case, the disturbance reflected from the pore bottom is in counter phase with the boundary-layer disturbance. However, the optimal thickness strongly depends on the disturbance frequency and the thick porous layer is more robust. Figure 8 illustrates the stabilization effect for three-dimensional waves of the second-mode family. The growth rate is shown as a function of the wave front angle $\varphi=\arctan \left(\beta_{r} / \alpha_{r}\right)$ at various pore radii. The porous coating causes massive reduction of the disturbance growth rate and substantially 


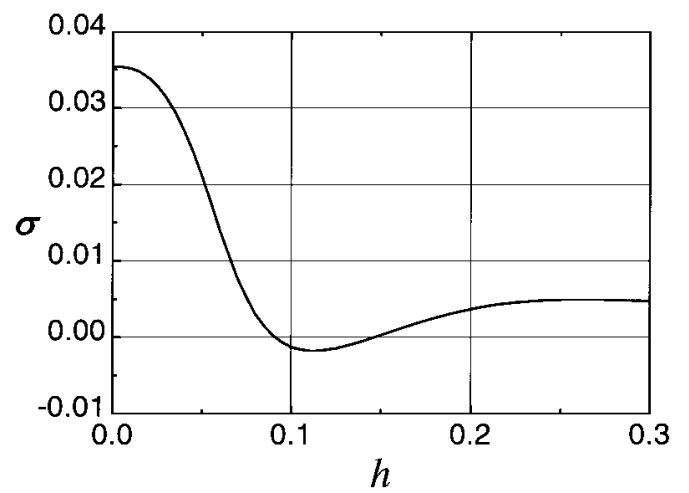

Fig. 7 Growth rate $\sigma$ as a function of porous layer thickness $h$ at $n=0.4, r=0.03, R e=4 \times 10^{3}, F=3 \times 10^{-4}, M=6$, and $T_{w}=1.4$ for zero thermal admittance.

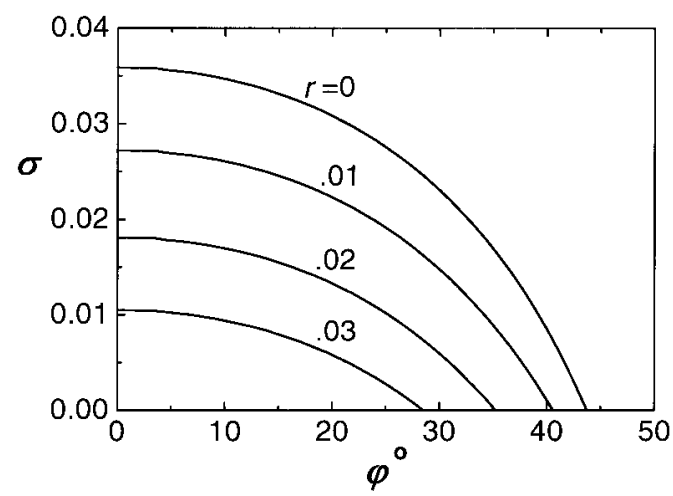

Fig. 8 Growth rate $\sigma$ as a function of wave front angle $\varphi$ at various pore radii $r: M=6, T_{w}=1.4, R e=4287, F=3 \times 10^{-4}, n=0.3$, and $\Lambda h \rightarrow \infty$.

decreases the unstable range of wave front angles. These examples show that a relatively thin porous coating can dramatically reduce the second-mode amplification and increase the laminar run if transition is driven by second-mode disturbances.

\section{Experimental Validation of Theory}

Rasheed et al. ${ }^{15}$ have recently verified the theoretical concept by testing a 5-deg half-angle sharp cone with an ultrasonically absorptive coating in the California Institute of Technology T5 highenthalpy shock tunnel. The cone was $1 \mathrm{~m}$ in length, with half of its surface solid and the other a porous sheet perforated with equally spaced blind cylindrical holes. Porosity parameters were chosen from the preliminary theoretical analysis of Fedorov and Malmuth as well as manufacturing constraints. The average pore radius $r^{*}$ was $30 \mu \mathrm{m}$, the depth $h^{*}$ was $500 \mu \mathrm{m}$, and the average spacing $s^{*}$ was $100 \mu \mathrm{m}$. Figure 9 shows a microphotograph of a portion of the porous surface. For typical runs, the boundary-layer thickness was about $1 \mathrm{~mm}$, and the estimated number of holes per boundarylayer disturbance wavelength was about 20. Static measurements of ultrasound reflectivity of perforated sheet coupons (without flow) showed that the porous coating attenuated the incident ultrasonic signal of $400-\mathrm{kHz}$ frequency by $3.0 \mathrm{~dB}$ relative to a solid wall.

The model was instrumented by thermocouples, and the transition onset point was determined from the Stanton number distributions $S t(x)$ measured simultaneously on both sides of the model for each run. Nitrogen was selected as the test gas to minimize the chemistry effects, which were not included in the theoretical analysis. Runs were performed for the ranges of the freestream total enthalpy $4.18 \leq H_{0} \leq 13.34 \mathrm{MJ} / \mathrm{kg}$ and Mach number $4.59 \leq M_{\infty} \leq 6.4$. Figure 10 shows a summary plot of the transition onset Reynolds number $R e_{\mathrm{tr}}=x_{\mathrm{tr}}^{*} U_{e}^{*} \rho_{e}^{*} / \mu_{e}^{*} \mathrm{vs} H_{0}$. The solid squares correspond to transition on the solid wall, and the open circles correspond to transition on the porous surface. The circles with arrows indicate that the boundary layer on the porous surface was

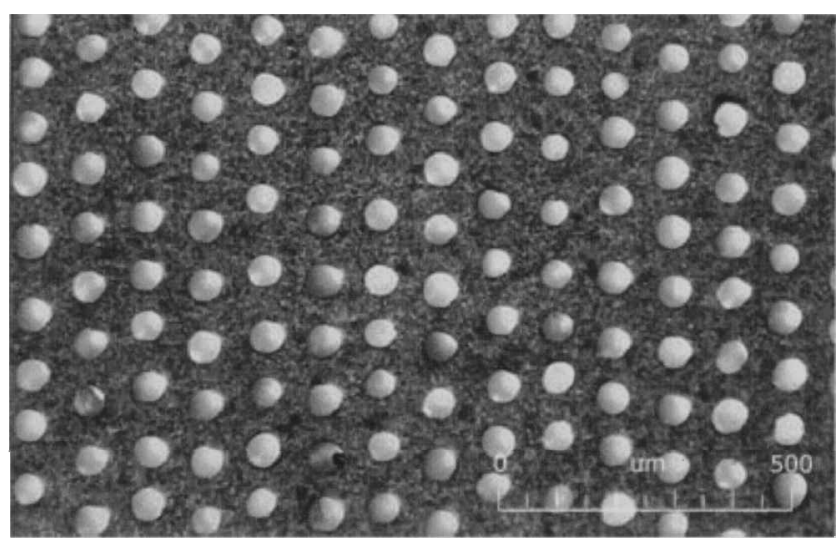

Fig. 9 Microphotograph of porous surface.

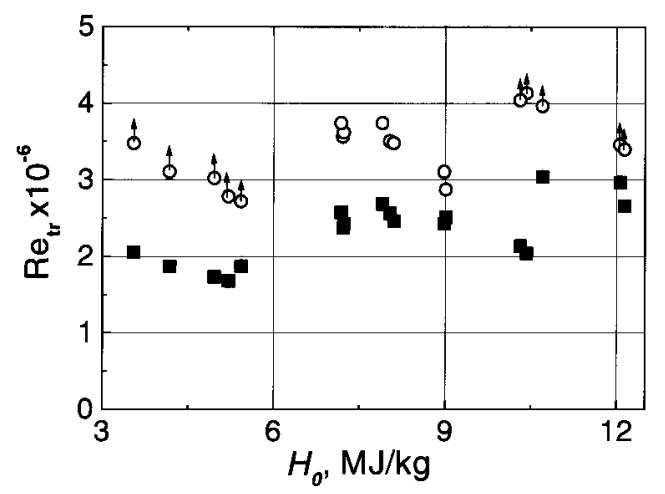

Fig. 10 Transition onset Reynolds number $R e_{\text {tr }}$ vs total enthalpy $H_{0}$ : $\square$, solid wall; $\circ$, porous wall; and $\delta$, boundary layer on porous wall is laminar up to the model base.

laminar up to the model base, that is, the value plotted is not a real data point because the cone was not long enough to measure the transition locus. In all cases, the circles are well above the squares. This indicates that the porous coating always delays transition by a significant amount.

\section{Summary}

A second-mode stability analysis has been performed for hypersonic boundary layers over walls covered by porous coatings with equally spaced blind microholes. Absorption of the disturbance energy by porous layers was modeled using the theory of disturbance wave propagation in thin and long tubes. The admittance and thermal admittance coupling the pressure disturbance with the vertical velocity and temperature disturbances on the porous surface are expressed as explicit functions of porosity characteristics. Stability calculations showed that the absorption of disturbance energy by the porous coating provides massive reduction of the secondmode growth rate in a wide range of disturbance frequencies and Reynolds numbers. The flow stabilization is due to vertical velocity perturbations on the porous surface associated with nonzero admittance of porous medium. Temperature perturbations weakly affect the boundary-layerdisturbance and can be neglected. This indicates that temperature disturbancesplay a passive role in the second-mode instability mechanism.

Our conclusions are consistent with the results of Malmuth et al., ${ }^{14}$ obtained from their inviscid stability analysis. The most profound effect is observed on a cool wall that is typical for hypersonic vehicle TPS surfaces. A relatively thin porous coating (of thickness about $30 \%$ of the laminar boundary-layer displacement thickness) provides a strong stabilization effect. Such porous coatings can be designed for passive LFC in hypersonic vehicle surfaces. Note that the disturbance absorption should be introduced at the initial phase of transition process, where the unstable disturbance amplitude is about $0.01-0.1 \%$ of its level in transitional and turbulent boundary layers. In this phase, additional heating of the porous coating 
associated with partial absorption of the disturbance energy is negligibly small compared to the turbulent heating.

The first series of experiments conducted by Rasheed et al. ${ }^{15}$ on a sharp cone in the T5 shock tunnel at the Graduate Aeronautical Laboratories at the California Institute of Technology qualitatively confirms the theoretical prediction. Quantitative comparison of the theory with these data is planned for the future. Because the boundary-layerstabilization is due to the disturbanceenergy extraction mechanism, we believe that similar effects may occur for other types of high-frequency instabilities. Absorptive coatings may also affect the bypass mechanism, which is responsiblefor transitionpast TPS roughness elements. These assumptions could be examined by further theoretical modeling and verified by experiments.

Many TPS materials, which can provide efficient absorption of acoustic disturbances, have a random porosity. The interaction of the boundary layer and unstable disturbances with a random porous coating may be different from the case of the regular pore structure discussedearlier. Because of communicationbetween randomly distributed pores, a mean flow may occur inside the coating that leads to a slip effect on the coating surface. Boundary conditions for unstable disturbances may be also affected. These effects will also be addressed in our future studies.

\section{Acknowledgments}

Portions of this effort was supported by the Air Force Office of Scientific Research, Air Force Materials Command under Contracts F49620-92-C-0006, F49620-96-C-0004, and F499620-98-10353. The U.S. government is authorized to reproduce and distribute reprints for government purposes, notwithstanding any copyright notation thereon. The views and conclusions herein are those of the authors and should not be interpreted as necessarily representing the official policies or endorsements, either expressed, or implied by the Air Force Office of Research or the U.S. government.

\section{References}

${ }^{1}$ Malik, M. R., Zang, T. A., and Bushnell, D. M., "Boundary-Layer Transition in Hypersonic Flows," AIAA Paper 90-5232, 1990.

${ }^{2}$ Reed, H. L., Kimmel, R., Schneider, S., and Arnal, D., "Drag Prediction and Transition in Hypersonic Flow," AIAA Paper 97-1818, June 1997.

${ }^{3}$ Bowcutt, K. G., Anderson, J. D., and Capriotti, D., "Viscous Optimized Hypersonic Waveriders," AIAA Paper 87-0272, 1987.

${ }^{4}$ Reshotko, E., "Boundary-Layer Instability, Transition, and Control," AIAA Paper 94-0001, Jan. 1994.

${ }^{5}$ Mack, L. M., "Boundary-Layer Stability Theory," Special Course on Stability and Transition of Laminar Flow, edited by R. Michel, Rept. 709, AGARD, 1984, pp. 3-1-3-81.

${ }^{6}$ Reshotko, E., "Stability Theory as a Guide to the Evaluation of Transition Data," AIAA Journal, Vol. 7, No. 6, 1969, pp. 1086-1091.

${ }^{7}$ Malik, M. R., "Prediction and Control of Transition in Supersonic and Hypersonic Boundary Layers," AIAA Journal, Vol. 27, No. 11, 1989, pp. 1487-1493.

${ }^{8}$ Malik, M. R., "Stability Theory for Laminar Flow Control Design," Viscous Drag Reduction in Boundary Layers, edited by D. M. Bushnell and J. N. Hefner, Vol. 123, Progress in Astronautics and Aeronautics, AIAA, Washington, DC, 1990, pp. 3-46.

${ }^{9}$ Kendall, J. M., "Wind-Tunnel Experiments Relating to Supersonic and Hypersonic Boundary-Layer Transition," AIAA Journal, Vol. 13, No. 3, 1975, pp. 290-299.

${ }^{10}$ Demetriades, A., "Hypersonic Viscous Flow over a Slender Cone, Part III: Laminar Instability and Transition,” AIAA Paper 74-535, 1974.

${ }^{11}$ Stetson, K. F., Thompson, E. R., Donaldson, J. C., and Siler, L. G., "Laminar Boundary-Layer Stability Experiments on a Cone at Mach 8, Part 1: Sharp Cone," AIAA Paper 83-1761, 1983.

${ }^{12}$ Stetson, K. F., and Kimmel, R. G., "On the Breakdown of a Hypersonic Laminar Boundary Layer," AIAA Paper 93-0896, 1993.

${ }^{13}$ Kimmel, R., Demetriades, A., and Donaldson, J., "Space-Time Correlation Measurements in a Hypersonic Transitional Boundary Layer," AIAA Paper 95-2292, 1995.

${ }^{14}$ Malmuth, N. D., Fedorov, A. V., Shalaev, V., Cole, J., and Khokhlov, A., "Problems in High-Speed Flow Prediction Relevant to Control," AIAA Paper 98-2995, June 1998.

${ }^{15}$ Rasheed, A., Hornung, H. G., Fedorov, A. V., and Malmuth, N. D., "Experiments on Passive Hypervelocity Boundary-Layer Control Using a Porous Surface," AIAA Paper 2001-0274, Jan. 2001.

${ }^{16}$ Mack, L. M., “Boundary-Layer Stability Theory," Jet Propulsion Lab., Rept. 900-277, rev. B, California Inst. of Technology, Pasadena, CA, 1969.

${ }^{17}$ Gaponov, S. A., "Influence of Porous Layer on Boundary-Layer Stability," Izevestia SO AN SSSR, Seria Technicheskich Nauk, Vyp. 1, No. 3, 1971, pp. 21-23 (in Russian).

${ }^{18}$ Gaponov, S. A., "Influence of Gas Compressibility on Stability of Boundary Layer on Porous Surface at Subsonic Speeds," Zhurnal Prikladnoi Mechaniki i Technicheskoi Fiziki, No. 1, 1975, pp. 121-125 (in Russian).

${ }^{19}$ Gaponov, S. A., "Stability of Supersonic Boundary Layer on Porous Wall with Heat Conductivity," Izvestia AN SSSR, Mechanika Zhidkosti i Gaza, No. 1, 1977, pp. 41-46 (in Russian).

${ }^{20}$ Stinson, M. R., and Champoux, Y., "Propagation of Sound and the Assignment of Shape Factors in Model Porous Materials Having Simple Pore Geometries," Journal of the Acoustical Society of America, Vol. 91, No. 2, 1992, pp. 685-695.

${ }^{21}$ Rzhevkin, S. N., Lectures on Theory of Sound, Moscow State Univ., Moscow, 1960 (in Russian).

${ }^{22}$ Daniels, F. B., "On the Propagation of Sound Waves in a Cylindrical Conduit," Journal of the Acoustical Society of America, Vol. 22, No. 2, 1950 , pp. 563-564.

${ }^{23}$ Benade, A. H., "On the Propagation of Sound Waves in a Cylindrical Conduit," Journal of the Acoustical Society of America, Vol. 44, No. 2, 1968, pp. 616-623.

${ }^{24}$ Malmuth, N., "Stability of the Inviscid Shock Layer in Strong Interaction Flow over a Hypersonic Flat Plate," Fluid Mechanics and Its Applications, edited by D. E. Ashpis, T. B. Gatski, and R. Hirsch, Kluwer Academic, Boston, 1993, pp. 189-223. 